\title{
A Test Pattern for Quality Control of Laser Scanner and Charge-Coupled Device Film Digitizers
}

\author{
Ethan J. Halpern
}

\begin{abstract}
Although clinical images provide the ultimate test of diagnostic performance for a film digitizer, such images are not generally suitable for daily quality control (QC) purposes. However, a well-designed test pattern will provide a rapid, comprehensive, objective and reproducible assessment of image quality. This pattern should evaluate various parameters of image quality, including high contrast resolution, low contrast discrimination, linearity of gray scale, geometric distortion, and noise. Furthermore, the pattern should detect light leak and film slippage, two problems commonly associated with film digitizers. The test pattern described in this manuscript was designed to provide quantitative measures of performance for a film digitizer. As part of a regular $Q C$ routine for a laser scanner or charge-coupled device digitizer, this pattern provides a simple method to identify and quantify changes in digital image quality.

Copyright $(1995$ by W.B. Saunders Company
\end{abstract}

KEY WORDS: picture archiving and communication systems (PACS), teleradiology, quality control, digitalized images.

$\mathbf{L}$ ASER SCANNERS and charge-coupled device (CCD) scanners are often used to digitize conventional analog radiographs for digital storage, transmission, or display. Laser scanners generally provide a greater dynamic range than CCD devices in terms of gray scale, but their cost is also considerably higher. Both laser scanners and CCD scanners include electronic hardware and mechanical parts that must be properly calibrated and which should be monitored with a regular quality control (QC) routine.

The performance of a film digitizer may be evaluated with either clinical images or a test pattern. Clinical images must be digitized from conventional radiographs and displayed on a monitor or printed to film; the digital image is then compared with the original radiograph. Such a comparison is, by its nature, qualitative and subjective. Furthermore, the observed image quality depends upon the digitizer, the display device, and the link between the two. Test patterns for film digitizers are provided on a film that must also be digitized; QC evaluation is then performed either directly on the digital data-a direct quantitative approach-or by evaluation of the digitized image on a video display-a semiquantitative approach. In contrast with the clinical image, the test pattern is designed to detect problems with image quality and to quantify the extent of those problems. Thus, a test pattern printed on a single film provides an evaluation of many different aspects of image quality that may not be adequately tested even with a large number of clinical images.

The Society of Motion Picture and Television Engineers (SMPTE) has developed a test pattern which is widely used to calibrate and test display monitors. ${ }^{1,2}$ This pattern provides line pairs to test high contrast resolution, squares of varying optical density to test gray-scale response, and a grid pattern to detect geometric distortion. Although this pattern is very useful for television monitors, it lacks several features which should be included to test radiologic image display systems. (1) The line pairs in this pattern are oriented in orthogonal axes and include a very limited range of line-pair densities. Off-axis resolution (ie, along a diagonal) is not tested. (2) The pattern provides only a rudimentary test for low contrast discrimination with $5 \%$ and $95 \%$ squares within the $10 \%$ and $100 \%$ squares. Low contrast resolution cannot be quantified with this pattern. (3) The grayscale response is tested with relatively large squares located in the center of the image; the pattern does not test for variance in gray-scale response that may be present from one side of the scanner to the other. (4) There are no specific tests for light leak or film slippage.

The test pattern described in this manuscript was designed specifically to evaluate laser scanner and CCD film digitizers. This pattern represents a recent modification of a previously described pattern that has been in use for

From the Department of Radiology, Jefferson Medical College of Thomas Jefferson University, Philadelphia, PA.

Address reprint requests to Ethan J. Halpern, MD, Department of Radiology, Thomas Jefferson University Hospital, 132 S 10th St, Philadelphia, PA 19107-5244.

Copyright $₫ 1995$ by W.B. Saunders Company

0897-1889/95/0801-0004\$3.00/0 
several years. ${ }^{3}$ The pattern provides tests for high contrast resolution, low contrast discrimination, linearity of gray-scale response, reproduction of continuous fine lines, geometric distortion and noise. A thin black border has been added around the entire pattern to detect light leak. Long columns of diagonally oriented lines have been added to detect any irregularity in movement of the film during digitization.

\section{TEST PATTERN DESIGN}

Figure 1 presents a photograph of the entire test pattern as well as detailed images of specific components within the pattern. The test pattern is defined by a digital data file with a resolution of 2,000 $\times 2,500$ pixels. This 10 -Mbyte data file is created by a $C$ language program, and is printed on a standard 14- $\times 17$-in film with a laser printer (Eastman Kodak, Rochester, NY) attached to a LINX system (DuPont Medical Products, Wilmington, DE). Printing parameters are adjusted for the smooth interpolation mode with the optical density range set to its maximum. The maximum pixel density on the pattern in both the horizontal and vertical axes is 3.5 line pairs ( $\mathrm{Ip}) / \mathrm{mm}$ (or 7 pixels $/ \mathrm{mm}$ ); this limitation is a direct result of the digital printing method. Although this resolution is sufficient for most teleradiology applications, selected applications such as mammography will require a test pattern with higher resolution. The optical density on the pattern has a range of 0.2 to 3.0 optical density units. The optical density of any individual area on the pattern will not generally vary by more than 0.1 optical density units from film to film. However, if the precise optical densities are required, the individual test film must be calibrated with a densitometer.

High contrast resolution is tested with line pairs oriented parallel to the margins of the film. Six sets of line pairs are provided with densities ranging from 0.6 to $3.5 \mathrm{lp} / \mathrm{mm}$. At 0.6 $\mathrm{lp} / \mathrm{mm}$, the optical densities of the light and dark portions of the line pairs are approximately 0.2 and 3.0 optical density units, respectively. As the line pair density approaches 3.5 $\mathrm{lp} / \mathrm{mm}$, the difference in optical density between the light and dark portions of the line pairs is minimally reduced, but the line pairs remain clearly defined on the test film. The placement of these line pair patterns along the periphery of the test pattern provides a simple test for barrel and pin cushion types of distortion on the digital display. The highest density line pairs are placed at the margins of the pattern to measure resolution where it is most likely to stress the system. High contrast resolution is tested along diagonal axes across the center of the pattern with line pair densities ranging from 0.6 to $2.6 \mathrm{lp} / \mathrm{mm}$. In addition, diagonally oriented high contrast line pairs are present in narrow columns along both sides of the pattern. Although these line pairs may also be used to test resolution, their primary purpose is to detect problems with the stepping motor or film slippage. When the film does not move smoothly through the digitizer these diagonal lines will not appear straight on the digitized image.

Low contrast resolution is tested with columns of small squares ranging in size from 0.1 to $1.6 \mathrm{~mm}$ embedded within a background whose optical density is $\sim 9 \%$ different than that of the squares. The low contrast pattern provides 16 columns with 11 squares in each column. In the 8 columns on the left side of the pattern, the squares are darker than their background; the remaining 8 columns contain squares that are lighter than their background. The background shades vary from 0.2 to 2.5 optical density units on the left side of the pattern, and 0.3 to 2.8 optical density units on the right side of the pattern.

Gray-scale response is tested with both continuous ramps and step patterns. Each step pattern contains 16 steps with optical densities ranging from 0.2 to 3.0 optical density units. Four large gray-scale patterns are provided to test the gray-scale response along the sides and in the center of the image. Twenty additional smaller 16-step gray-scale strips are provided to test for variance in the digitizer's gray-scale response across the digitized image. A 16-step gray-scale pattern was chosen by analogy with a recent report suggesting that 16 steps should be used to test film density in computed radiography systems. ${ }^{4}$ In addition to its quality control (QC) function, the gray-scale step patterns may also be used to adjust the output lookup table associated with the film digitizer to the desired gamma curve. ${ }^{5}$

Sets of three fine continuous vertical lines are 

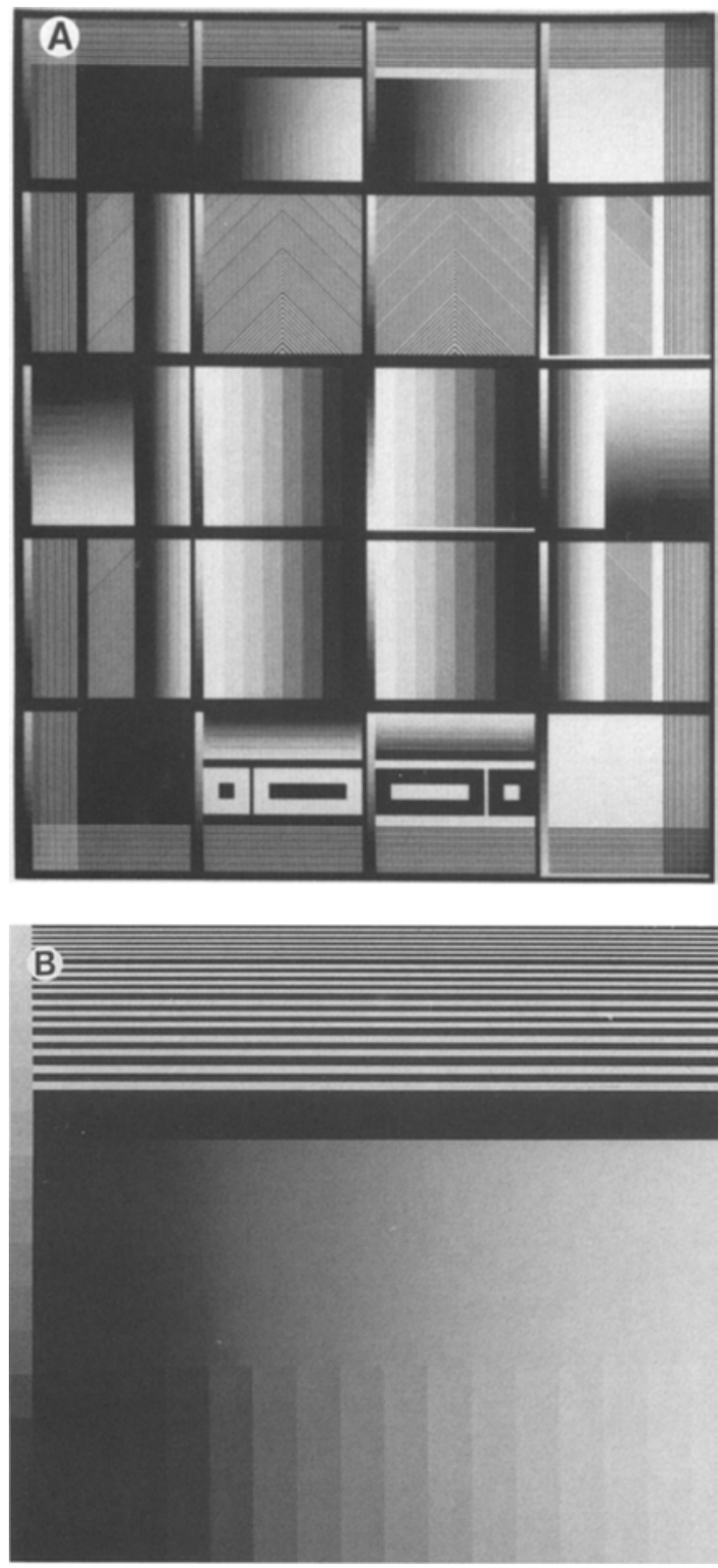

Fig 1. (A) Test pattern for film digitizer OC. (B) Close-up view of the test pattern showing the horizontal line pairs, a continuous gray-scale ramp and a gray-scale step pattern with 16 steps. (C) Close-up view of the test pattern showing the diagonal line pairs. Printing the pattern in smooth interpolation mode provides smooth diagonal line pairs. Diagonal lines will appear jagged in the sharp interpolation mode. (D) Close-up view of the test pattern showing the test for low-contrast discrimination. The embedded squares in each column become progressively smaller toward the top of the column.
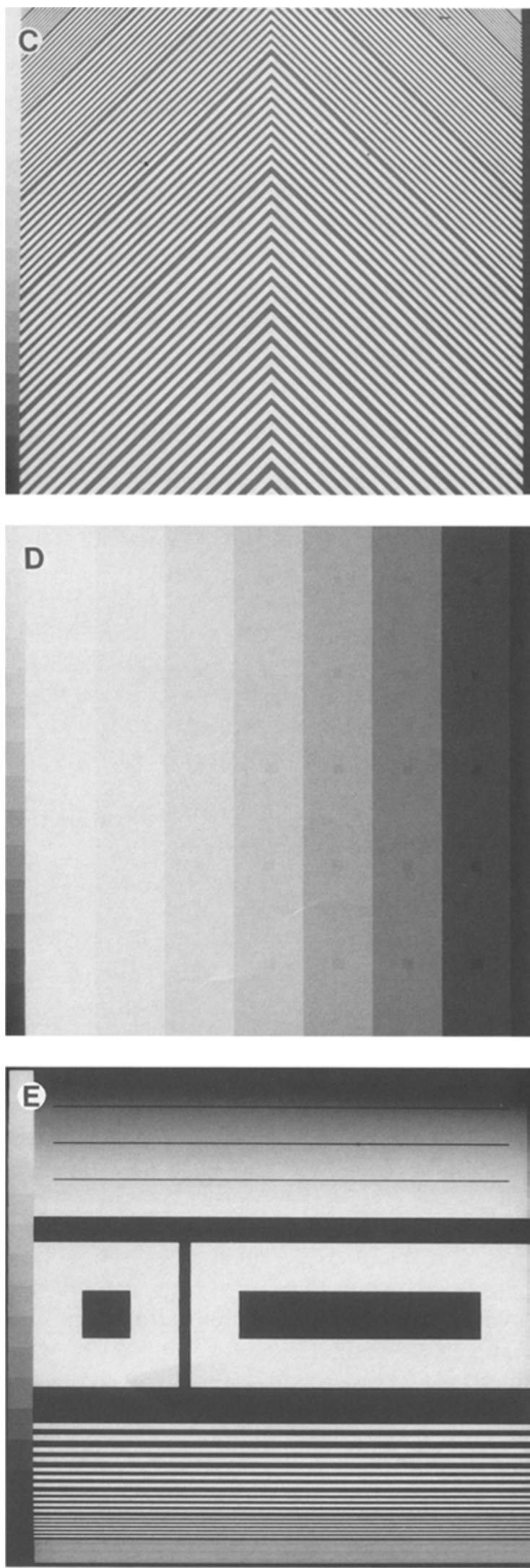
present along either side of the pattern embedded in a background of varying optical density. Two such sets of horizontally oriented lines are present along the bottom the pattern. These patterns test the ability of the film digitizer to reproduce a fine continuous line.

Geometric distortion is tested throughout the image. The pattern is organized into 20 square units that should appear square in the digitized image. Furthermore, the high contrast line pairs should intersect at 90 degrees in the corners of the pattern. Finally, there are smaller bright and dark squares embedded inside other squares at the bottom of the pattern. These squares and the adjacent rectangles with embedded rectangles also serve to detect streaking or blooming that may be present in the digitized image at interfaces of very bright and dark densities.

At the four corners of the pattern there are bare areas that may be used to test for noise in the digitized image. These areas appear relatively homogeneous on the test film. The digitized image should present these areas with a similarly homogeneous texture.

Finally, there is a thin black border around the entire pattern. Any leakage of light into the digitizer will present as a bright area within this border on the digitized image.

\section{TESTING A FILM DIGITIZER}

Once the test film has been digitized, it should be displayed at full resolution on a display monitor. When necessary, portions of the image should be magnified and the window and level settings should be adjusted to eliminate any loss of resolution or dynamic range caused by the display system. The test pattern may be used for QC testing of a laser scanner or CCD film digitizer with either a semiquantitative or a quantitative approach. This section outlines the procedures for each of these approaches. For daily QC monitoring of a film digitizer, the semiquantitative approach (with simple visual inspection of the digitized image) should suffice; the results should be recorded in a $\log$ book (Fig 2) and compared with previous results. However, when there is a need for more rigorous testing the digitized data should be analyzed to provide quantitative measures of image quality.

\section{High Contrast Resolution}

Note the number of line pair sets that are clearly defined in each of the horizontal, vertical, and diagonal axes. For quantitative measurement of high contrast resolution: Create a histogram along a line drawn perpendicular to the line pairs in the digitized pattern. The histogram will display a sinusoidal curve of increasing and decreasing digital pixel values (Fig 3). This curve provides an approximation of the MTF function of the digitizer. The limiting resolution may be defined as the point where the excursion of the sinusoidal curve is one half (or one third, etc) of its original amplitude.

\section{Low Contrast Discrimination}

Note the number of low contrast squares visible in each column. For quantitative measurement of low contrast discrimination, create a histogram along a line connecting the 11 low contrast squares in each column. The histogram should display a change in digital pixel value over each square. The limiting resolution may be defined by the square where the change in digital pixel value is one half (or one third, etc) of that seen in the largest square.

\section{Gray-Scale Response}

Each of the 16 steps in each of the step patterns should be visibly distinct from the adjacent steps. For quantitative gray-scale response, create a histogram of pixel values along the gray-scale ramps and step patterns. The mean digital pixel value corresponding to each step in the step pattern should be plotted against the known optical density of that step on the film to show the gamma function of the digitizer (Fig 4). The variance of the digital values within each step can be plotted against the optical density of that step to establish a relationship between noise in the digitized image and optical density. This may provide an important parameter for lesion detectability within backgrounds of varying optical density.

\section{Fine-Line Reproduction}

Each of the fine lines in the test pattern should be present and continuous in the digital display. On many systems the lines will appear to fade in and out at regular intervals. 
System

High contrast discrimination

(record \# of line pair groups visible)

horizontal

vertical

diagonal

Low contrast discrimination

(record \# of embedded boxes visible)

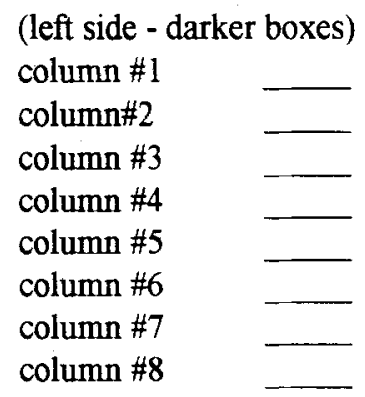

(right side - lighter boxes)

column \#1

column\#2

column \#3

column \#4

column \#5

column \#6

column \#7

column \#8

Gray scale response

(record \# of steps visible in 16 step patterns)

left side

top left

top right

right side

Fine line visibility

(record discontinuities in digitized fine lines)

Stepping motor reliability

(note discontinuities in the diagonal line pairs)

Date 


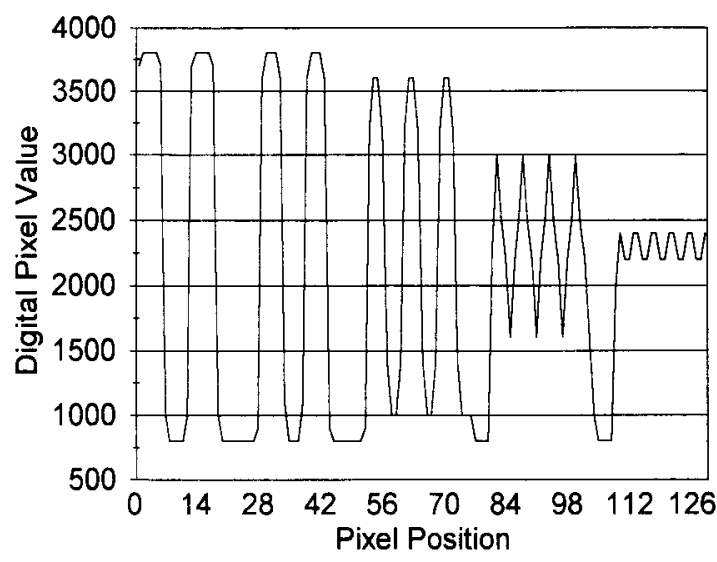

Fig 3. Histogram of digitized image data along a line perpendicular to a line-pair pattern. The excursion of the curve decreases when the line pairs are denser. The limiting resolution may be defined as the line-pair set at which the amplitude of the sinusoidal curve is one half of its original amplitude. For the current example, this would correspond to the fourth set of line pairs from the left side.

\section{Geometric Distortion}

Square areas on the test image should appear square on the display. For quantitative measurement of geometric distortion, measure the dimensions of each of the 20 square units in the pattern. The sides of the squares should be equal in length if the aspect ratio in the digitized image is correct. The diagonal measurements across each square unit should be equal unless there is loss of orthogonality. Small amounts of geometric distortion may be difficult to appreciate without such measurements.

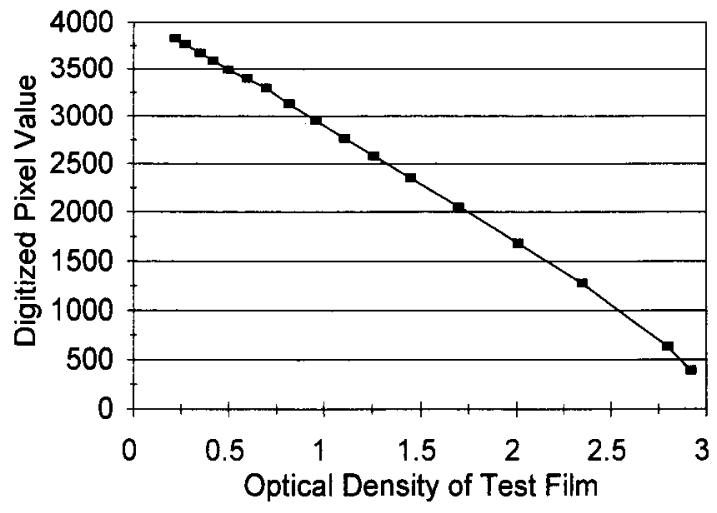

Fig 4. Plot of digital pixel value against optical density of the step pattern on film. This plot may show a linear, logarithmic, or other complex relationship depending upon the physical characteristics of the digitizer and the lookup table used for digitization.
Noise

The bare areas in the corners of the pattern should appear homogeneous on the digital display. For quantitative measurement of noise, define a region of interest within each bare area. The standard deviations of the digital pixel values within these regions provide a measure of noise. As mentioned above, noise may also be computed along the gray-scale steps to show the relationship between noise and optical density.

\section{Film Movement}

The diagonal lines in the test pattern should appear straight on the digital display. Any change in the rate at which the film moves through the digitizer will be seen as a nonlinear segment within the diagonal line pattern.

\section{Light Leakage}

Check the black border around the pattern for any light areas.

\section{DISCUSSION}

Picture archiving and communication systems (PACS) are used to store, transmit, and display radiographic images for diagnostic purposes. As such, the diagnostic performance of these systems in the clinical setting provides the most appropriate and, ultimately, the most important measure of image quality. For this reason, many well-controlled multi-observer studies have been performed to compare digital and conventional image displays for the diagnosis of various forms of clinical pathology. ${ }^{6-9}$ Similarly, PACS vendors often use diagnostic images to show or test their systems. Radiologists who purchase these PACS systems may also view a small set of clinical images to form their own assessments of image quality.

However, a single observer with selected diagnostic images provides a subjective and incomplete evaluation of image quality for a film digitizer. Radiographic images must be evaluated in a controlled environment where they are presented to multiple observers as randomized pairs to detect subtle differences in image quality. A single individual looking at a digital image display may not notice subtle changes in image quality. ${ }^{10}$ Furthermore, clinical images are often presented with a defined 
task of limited scope. When a chest radiograph is evaluated for the presence of interstitial lung disease, the ability of the system is tested with respect to its limits of resolution. To identify pulmonary nodules, a system must provide adequate low-contrast discrimination. The diagnosis of pneumothorax may depend on the ability of a system to reproduce continuous fine lines. Furthermore, two independent observers may concentrate on different aspects of the image and reach entirely different conclusions regarding image quality. Given an abdominal flat plate, one observer may concentrate on the visibility of a small calcification (high-contrast resolution) while a second observer concentrates on the detectability of a renal mass (low-contrast discrimination).

In contrast with clinical images, the test pattern presented in this manuscript allows a quick and objective evaluation of image quality by a single observer. For the nonradiologist, the pattern has an obvious advantage over clinical test images. However, even the sharp eyes of a radiologist may not appreciate the presence of subtle problems with image quality on clinical images. The test pattern includes multiple subpatterns to evaluate specific parameters of image quality through either a semiquantitative approach (by visual inspection of the digital display) or a more rigorous quantitative analysis of digitized pixel values. As part of a regular QC routine, this pattern will assist in the detection of subtle problems with image quality and will provide objective evidence to document those problems that do exist. Copies of this test pattern are available from the author.

\section{REFERENCES}

1. Gray JE, Kiks KG, Haddick DH, et al: Test pattern for video displays and hard copy cameras. Radiology 154:519527,1985

2. Roehrig H, Blume H, Ji TL, et al: Performance tests and quality control of cathode ray tube displays. J Digit Imaging 3:134-145, 1990

3. Halpern EJ, Esser PD: An improved phantom for quality control of laser scanner digitizers in picture archival and communications systems. J Digit Imaging 4:241-247; 1991

4. Huda W, Jing Z, Hoyle BA: Film density calibration for computed radiography systems: Is the standard threepoint procedure accurate? Radiology 188:875-877, 1993

5. Siegel EL, Templeton AW, Cook LT, et al: Image calibration of laser digitizers, printers and gray scale displays. Radiographics 12:329-335, 1992

6. Ackerman SJ, Gitlin JN, Gaylor RW, et al: Receiver operating characteristic analysis of fracture and pneumonia detection: Comparison of laser-digitized workstation images and conventional analog radiographs. Radiology 186 : 263-268, 1993

7. Slasky SB, Gur D, Good WF, et al: Receiver operating characteristic analysis of chest image interpretation with conventional, laser printer and high resolution workstation images. Radiology 174:775-780, 1990

8. Sajardo LL, Hillman BJ, Pond GD, et al: Detection of pneumothorax: Comparison of digital and conventional chest imaging. AJR 152:475-480, 1989

9. Halpern EJ, Newhouse JH, Amis ES, et al: Evaluation of teleradiology for interpretation of intravenous urograms. J Digit Imaging 5:101-106, 1992

10. Good WF, Gur D, Feist JH, et al: Subjective and objective assessment of image quality-a comparison. J Digit Imaging 7:77-78, 1994 